\title{
Malaria vaccine not perfect, but still useful
}

$\mathrm{T}$

he first vaccine against malaria - and the first against any parasitic disease in humans - has limited efficacy in children of $27 \%$ to $39 \%$, but it's still a useful tool in combatting a long-standing and dire disease, say experts.

"The pattern of disease arising from malaria is still very high" despite measures such as insecticidal bed nets and clearing brush, which is why a vaccine is welcome, explains Dr. Githinji Gitahi, chief executive officer of Amref Health Africa, a humanitarian organization providing training and health services in more than 30 countries, including those most affected by malaria. In sub-Saharan Africa, an estimated $14 \%$ of children aged 2 to 10 had malaria in 2013, a prevalence substantially reduced since 2000 .

Mosquirix, manufactured by GlaxoSmithKline, received a positive scientific opinion from the European Medicines Agency (EMA) July 24 even though it provides only partial and time-limited coverage, especially in children, who are most at risk of death due to malaria. Sir Brian Greenwood, principal author of the phase III randomized controlled trial in infants and children in sub-Saharan Africa (published in The Lancet) that formed the basis of the EMA opinion, says his study's findings are "disappointing."

"It's not quite as big a success as we would have wanted," says Greenwood, a professor of clinical tropical medicine at the London School of Hygiene \& Tropical Medicine in London, UK. Vaccine efficacy was $39 \%$ in children 5 to 17 months old and $27 \%$ in infants 6 to 12 weeks old, and this required three doses of vaccine plus a booster dose 18 months later. Without a booster, efficacy was even lower, indicating that protection wanes quickly. In addition, the vaccine induces immunity only to malaria caused by the Plasmodium falciparum parasite, the main disease-causing organism in sub-Saharan Africa. A proportion of malaria elsewhere in the world is caused by other Plasmodium parasites against which the vaccine has no effect.

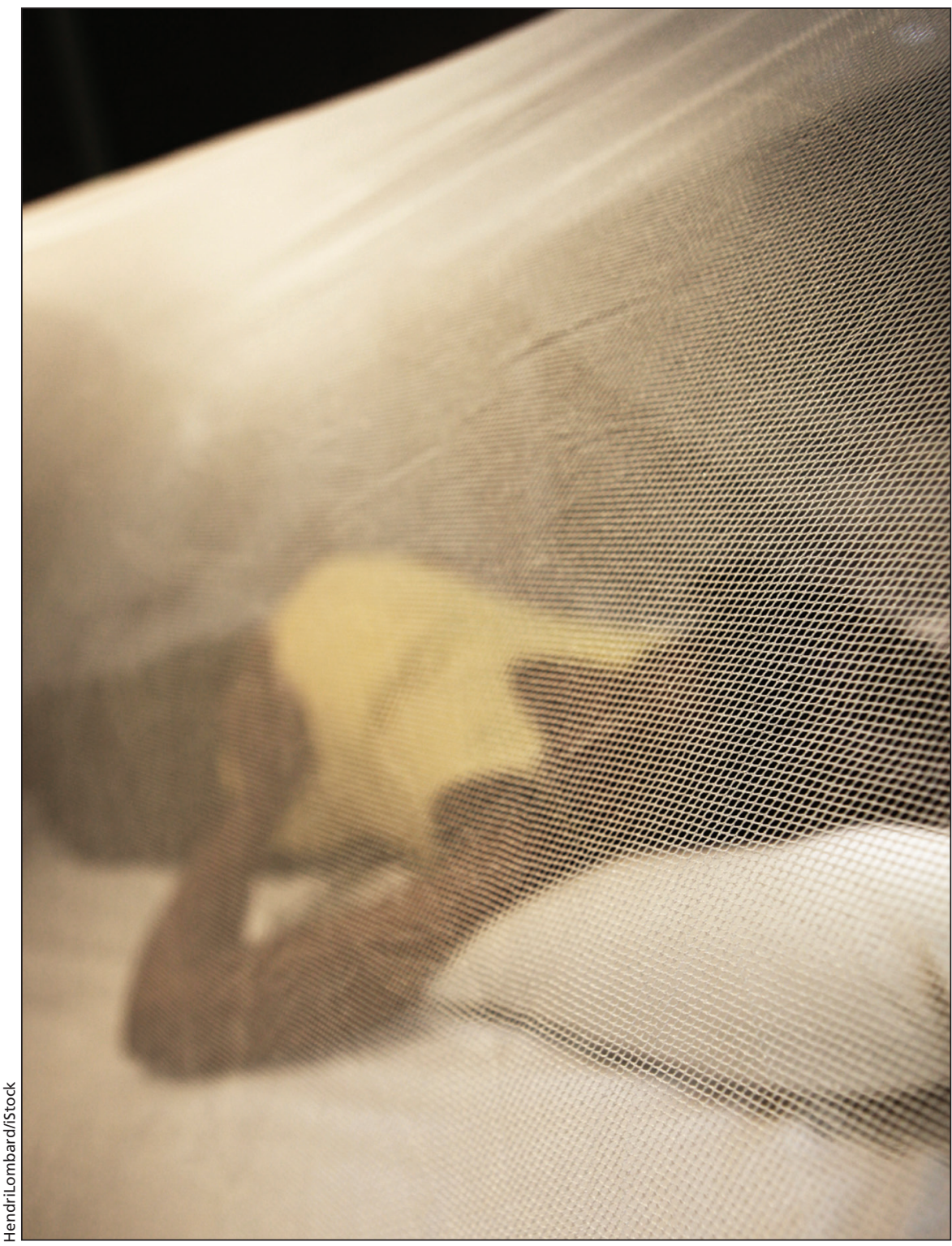

The vaccine alone isn't sufficient to control malaria; insecticidal bed nets and other measures must be retained.

Gitahi says the vaccine is useful nonetheless. "There is that kind of disappointment that the age and coverage is inadequate, but it is incremental. If we can save 1 million more lives through the vaccine, why not?"

Greenwood agrees, pointing out that "we use other vaccines that are not fully effective, such as the influenza vaccine." The overall effectiveness of the flu vaccine in 2013/14 in the US was $51 \%$ according to the US Centers for Disease Control and Prevention.

"Malaria is such a common problem that this [efficacy] makes it worth- while in some areas where infections are not very well controlled," says Greenwood.

In fact, finding a vaccine that works at all has been a long quest. Greenwood describes earlier efforts as cycles of hope, hype and disappointment. The difficulty is the nature of the disease itself. "The malaria parasite is so much more complicated than a bacteria or virus," he explains. "It has redundant mechanisms" that allow it to evade immune systems. "It's a much bigger challenge to develop an immune response." 
With EMA's opinion, the World Health Organization (WHO) will now convene its Strategic Advisory Group of Experts on Immunization and the Malaria Policy Advisory Committee to make a preliminary recommendation on whether and how to use the vaccine by the end of October; a final WHO policy recommendation is expected by November. Gitahi explains that malaria-affected countries then must provide national regulatory approval, which may be expedited through a WHO recommendation.

Procuring the vaccine is also a challenge for low-income countries. GlaxoSmithKline has promised to make the vaccine available at a reduced price although it hasn't set that price yet that would cover manufacturing costs plus a small return. Its profits will go back into research on vaccines for malaria and other tropical diseases. In addition, it will donate 12.5 million doses to PATH, an international nonprofit health organization that was a partner in developing the vaccine.

Careful planning of vaccine implementation in communities is the key to success, agree Gitani and Greenwood. Both are concerned that the introduction of a vaccine could lead to complacence about other prevention measures.

"Once the vaccine is available, governments and organizations like ours have to be very careful about the rollout," says Gitahi. "It should not be about the vaccine but about a two- or three-step 'this-is-how-you-preventmalaria' program. If you roll out [the vaccine] singularly, you could lose the gains that have been made with insecticidal nets."

Greenwood points out that, similarly, "in some of the HIV vaccine trials there was concern that people would stop using condoms, but that didn't happen." He thinks vaccine implementation requires "a proper community education program" that clearly informs those at risk, "Don't stop using your bed net."

Gitani hopes that use of the vaccine will lead to continued vaccine development. "Following precedent in medicine and research, once there is one, and once it is field-tested, the challenges are easier to see. In a few years there will probably be vaccines that are improvements over this one." - Carolyn Brown, Ottawa, Ont.

CMAJ 2015. DOI:10.1503/cmaj.109-5132

\section{Growing the evidence base for medical cannabis}

A necdotal reports of efficacy from patients who use medical marijuana aren't enough to convince many physicians that cannabis is a viable therapeutic option, but new industry-funded research in Canada may bring some science to the situation.

Some of Canada's 25 or so licensed producers under the federal medical marijuana program are investing in research to add credibility to their products. As of June 2015, Health Canada had approved six clinical trials involving patients who use dried cannabis; several others are pending approval. There are also a few large-scale observational studies, including a provincewide one in Quebec.

Medicinal marijuana is used for an array of symptoms and conditions, including multiple sclerosis, osteoarthritis, cancer and anxiety. It's up to the discretion of the physician to prescribe access, but the problem is the lack of rigorous guidelines based on scientific evidence.

Many licensed producers have been collecting patient data, and some are now partnering with university-based researchers to conduct large-scale observational studies; one wants to enroll 1000 patients, another is aiming for 3000 .

This research is possible, in part, because the growing industry now wants to show efficacy for its lucrative products - and is willing to pony up the cash to obtain evidence. Also, there are many more strains of cannabis to be tested than even two years ago. Prior to 2013, there vaporizer, as well as a placebo stripped of the two main active compounds, tetrahydrocannabinol and cannabidiol.

Leading the trial is Zach Walsh, the codirector for the Centre for the Advancement of Psychological Science and Law at UBC, who plans to recruit 40 patients who have served in the military or as first responders, or have survived a sexual assault. He says existing

\section{"We are catching up with patients who have been leading the medical cannabis movement."}

was one government-approved producer and one strain of the plant. Now there are hundreds of strains.

Tilray, for example, has 55 strains in its \$30-million facility in Nanaimo, British Columbia. Three of those strains will be used at the University of British Columbia (UBC) in Kelowna for what may become Canada's first randomized clinical trial on the use of cannabis for posttraumatic stress disorder (PTSD). Tilray is providing $\$ 400000$ and the dried bud for patients to use with a treatments for a large number of people with PTSD are not effective, and many are already using cannabis to ease their symptoms. "We are catching up with patients who have been leading the medical cannabis movement."

Tilray is "in a unique position to be able to fund and support this kind of research in a way that hasn't happened in North America in the past," says Philippe Lucas, the company's vice president of patient research and services. Tilray is exploring other oppor- 